\title{
Unifocal versus Multiple Ileal Neuroendocrine Tumors Location: An Embryological Origin
}

\author{
Maroin Kalifi ${ }^{a}$ Thomas Walter ${ }^{b, c, d}$ Laurent Milot ${ }^{c, d, e}$ Valérie Hervieu ${ }^{c, d, f}$ \\ Ingrid Millot ${ }^{g}$ Benjamin Gibert ${ }^{c, h}$ Colette Roche ${ }^{c}$ Julien Forestier ${ }^{b}$ \\ Catherine Lombard-Bohas $^{\mathrm{b}}$ Arnaud Pasquer ${ }^{\mathrm{a}}$ Gilles Poncet $^{\mathrm{a}, \mathrm{c}, \mathrm{d}}$
}

aHospices Civils de Lyon, Hôpital Edouard Herriot, Chirurgie Digestive, Lyon, France; ${ }^{b}$ Hospices Civils de Lyon, Hôpital Edouard Herriot, Service de Gastroentérologie et d'Oncologie Digestive, Lyon, France; CINSERM, UMR 1052UMR5286, UMR 1032 Lyon Cancer Research Center, Faculté Laennec, Lyon, France; 'Université de Lyon, Université Claude Bernard Lyon 1, Villeurbanne, France; eHospices Civils de Lyon, Hôpital Edouard Herriot, Radiologie, Lyon, France; ${ }^{\mathrm{f} H}$ ospices Civils de Lyon, Hôpital Edouard Herriot, Service Central d'Anatomie et Cytologie Pathologiques, Lyon, France; ${ }^{9}$ Hospices Civils de Lyon, Hôpital Édouard Herriot, Service d'anesthésie-réanimation, Lyon, France;

${ }^{\mathrm{h}} \mathrm{CNRS}$ Rhône Auvergne, Villeurbanne, France

\section{Keywords}

Small intestine $\cdot$ Surgery $\cdot$ Neuroendocrine tumors .

Multifocal · Location

\begin{abstract}
Introduction: Small-intestinal neuroendocrine tumors (SINET) are situated preferentially within the ileum. The aim was to describe a potential difference in location between unifocal and multiple ileal-NET. Patients and Methods: Between December 2010 and December 2019, all consecutive patients who underwent resection in our European Neuroendocrine Tumor Society Center of Excellence, of at least 1 non-duodenal SI-NET, were retrospectively included. The main objective was to prove that multiple ileal-NET were mostly located on the left side of the superior mesenteric artery (SMA) axis (defined as $40 \mathrm{~cm}$ from the ileocecal valve), and unifocal ones on the right side. Results: Ninety-four patients were included, 6 with unifocal jejunal-NET located 35
\end{abstract}

$\mathrm{cm}$ (range, 10-60) from the duodenojejunal angle (DJA), 44 $(47 \%)$ with unifocal ileal-NET and 44 (47\%) with multiple ileal-NET. The median number of tumors in multiple ileal-NET was 7 (range, 2-95), within a median small bowel segment of $105 \mathrm{~cm}$ (10-240). The median length between the proximal tumor and the DJA was $428 \mathrm{~cm}(300-635)$ and $540 \mathrm{~cm}$ (350-725) for the distal one; 40 (91\%) of them were located on the left side of the SMA axis. In contrast, unifocal ileal-NET were located at a median distance of $577 \mathrm{~cm}(305-820)$ from the DJA ( $p<0.001$, compared to multiple ileal-NET); $30(68 \%)$ of them were on the right side of the SMA axis $(p<0.001)$. Conclusion: Multiple ileal-NET are mostly located on the left side of the SMA axis. Further studies are warranted to explore the embryological origin of unifocal versus multiple ilealNET.

(c) 2020 S. Karger AG, Basel

Synopsis: Location of unifocal and multiple small-intestinal neuroendocrine tumors according to the superior mesenteric artery axis. karger@karger.com

(c) 2020 S. Karger AG, Basel

www.karger.com/nen

Karger
Thomas Walter

Gastroenterology and Medical Oncology, Hôpital Edouard Herriot

University Hospital of Lyon, 5, Place d'Arsonval

FR-69437 Lyon Cedex 03 (France)

thomas.walter@chu-lyon.fr 


\section{Introduction}

Small-intestinal neuroendocrine tumors (SI-NET) are rare, but their incidence has increased by $300-500 \%$ over the past 40 years [1]. SI-NET have the particularity of being multiple in $30-56 \%$ of cases [2-5]. There is currently no known pathogenetic mechanism underlying the development of multiple tumors, and prognosis is similar to that of unifocal SI-NET [4, 5]. Multiple SI-NET appear to develop in a limited segment of the small bowel (SB), $89 \%$ of them being located in the ileum, most often within 100 $\mathrm{cm}$ of the ileocecal valve (ICV) [6]. A difference in location between unifocal and multiple ileal-NET has never been described. The hypothesis made herein was that there could be an embryological origin for differential location: the superior mesenteric artery (SMA) could delineate the distribution of unifocal tumors to its right side versus multiple SI-NET to its left side. Indeed, the SMA constitutes the anatomic axis of the primitive gut $[7,8]$, which separates the future duodenum, jejunum, and a major part of the ileum on one side, and the last part of the ileum and the colon on the other side (Fig. 1). The terminal branch of the SMA crosses the ileum to supply the vitelline duct. During the fifth week of gestation, complete obliteration of the vitelline duct occurs. In 2 or $4 \%$ $[9,10]$, this obliteration is incomplete, and a blind intestinal pouch may persist: the Meckel's diverticulum (MD). Whether present or not, the theoretical area of the MD represents a base point along the SB to affirm whether one tumor is on one side or the other of the SMA. Although data from the literature are rather contradictory with respect to the median distance between the ICV and MD, the latter can be considered to be around $40 \mathrm{~cm}$ from the ICV $[9,11,12]$. We have therefore designed a retrospective study to explore the location of all removed SI-NET in relation to the SMA axis.

\section{Patients and Methods}

All consecutive patients who underwent resection of at least 1 primary SI-NET by a single surgeon (GP) between December 2010 and December 2019 in the Lyon European Neuroendocrine Tumor Society (ENETS) Center of Excellence have been included. Patients with a duodenal NET were not included. Patients who underwent prior surgical SB resection concerning SI-NET and those for whom SB measurements were not feasible were excluded.

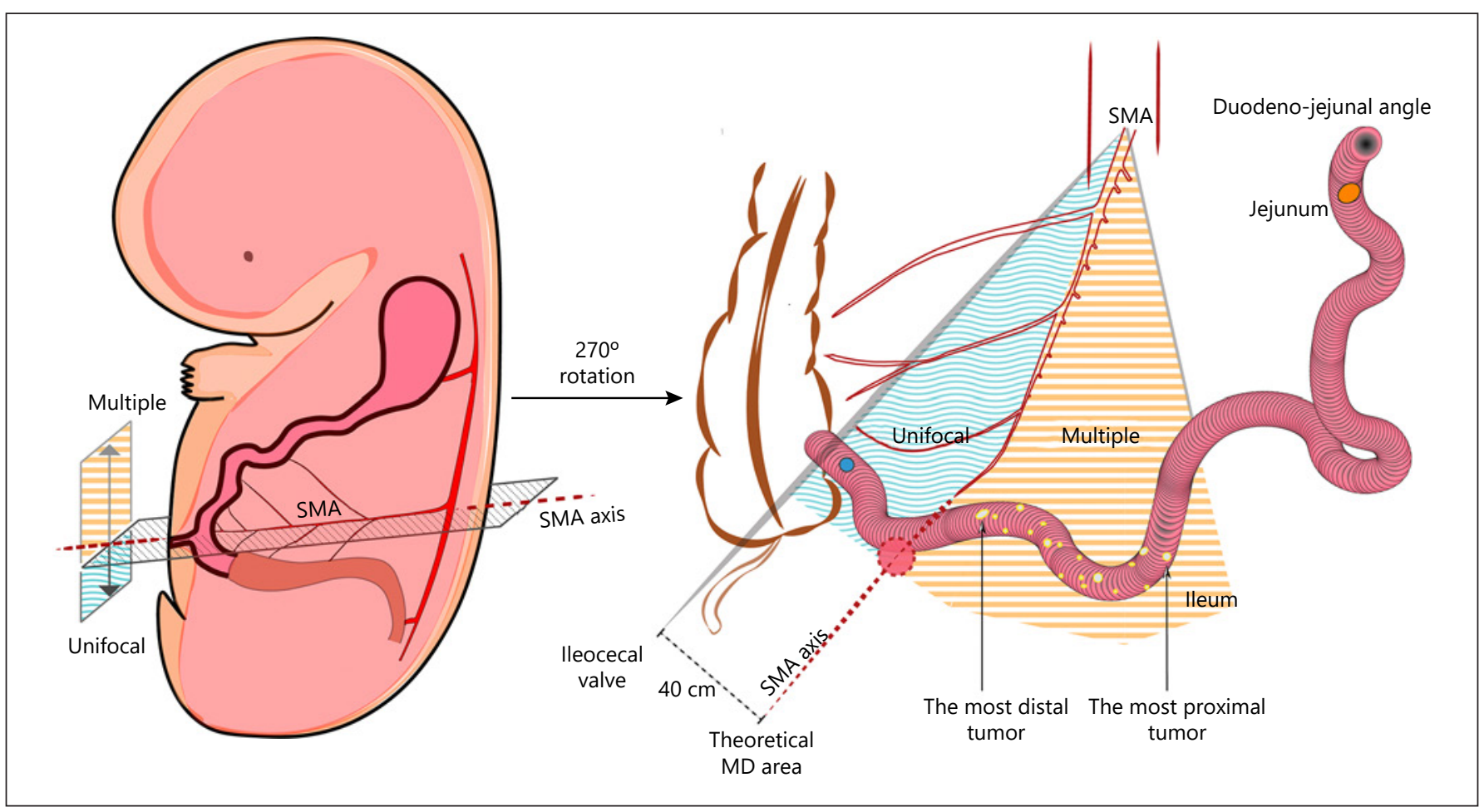

Fig. 1. SI-NET location according to the SMA axis. MD, Meckel's diverticulum; SI-NET, small-intestinal neuroendocrine tumors; SMA, superior mesenteric artery axe.

Small-Intestinal Neuroendocrine Tumors Location
Neuroendocrinology 2021;111:786-793 DOI: $10.1159 / 000511849$ 
The surgeon (GP) and an expert pathologist (VH) were both members of the French neuroendocrine national reference network [13-16]. Surgical resection consisted of a hemicolectomy and/or a SB resection, depending on tumor location and lymph node (LN) involvement [15]. Extensive lymphadenectomy was performed from the SB section to the retropancreatic area [5]. For each patient, measurements were based on the operative note and pathological reports, which are standardized and reproducible; by compression and bidigital palpation, the total length of the SB was routinely analyzed and each tumor was marked with a polypropylene suture [14]. We reported the total length of the SB, the number of tumors, the exact location from the duodenojejunal angle (DJA) for each tumor, and the total length between the last SI-NET from the ICV, between the first and the last SI-NET, and between each tumor. Tumor locations were also assessed as a percentage of the entire jejuno-ileum (JI) length from the DJA and from the ICV. All suspected SI-NET had to be confirmed by the pathologist to be counted; the location of missing tumor lesions was measured by the pathologist. Distant invasion of the liver or peritoneal metastases were also reported. We considered a patient to be symptomatic when in the presence of preoperative diarrhea, abdominal pain, anemia, or weight loss.

Samples were received fresh and were opened along the antimesenteric border. The specimen was examined macroscopically, and after fixation, all tumors were counted and sampled. The following information was collected for each tumor: maximum diameter, histological grade (according to the WHO 2019 classification), and the pTNM classification according to the 8th edition (2017) of the UICC cancer staging. The number of removed LNs, involved LN, and LN ratio were collected. All data from the operative report were then correlated with the final pathology report. SI-NET were then divided into 2 groups: unifocal and multiple SINET.

Categorical variables are presented as number (percentage), continuous variables as median (range). The $\chi^{2}$ or Fisher's exact tests were used to compare groups for qualitative variables, and a $t$ test was used for quantitative variables. Overall survival was estimated using the Kaplan-Meier method and was calculated from the date of the surgery, date of death, or last follow-up. Univariate analyses were performed using the Log-rank test for each variable of interest. All statistical analyses were performed using IBM-SPSS version 21 (IBM Corporation, Armonk, NY, USA).

\section{Results}

During the 10-year study period, 309 jejuno-ileal SINET were identified: 65 did not undergo SB resection, 131 were performed by a surgeon other than GP (the only surgeon who measured the exact required SB distances), and exact measurements for tumor localization were not possible in 19 patients. Therefore, 94 patients were studied: 6 patients (6\%) had unifocal jejunal-NET, $44(47 \%)$ had unifocal ileal-NET, and $44(47 \%)$ had multiple SI-NET. All 6 jejunal-NET were unifocal, located at a median distance of $35 \mathrm{~cm}$ (range, 10-60) from the DJA; their median size was $47 \mathrm{~mm}$ (range, 20-120),
5 were pT3, and their median Ki67 was 4\% (range, 1.430). The median age was 57 years (range, 45-70). Of these 6 patients with jejunal-NET, one had carcinoid syndrome; 3 had locoregional LN involvement, 5 had distant metastatic disease ( 4 in the liver and 1 in the peritoneum), and 3 died within 5 years after surgery ( 5 year survival was $50 \%$ ).

We focused the analysis on the 88 ileal-NET. The median age was 66 years (range, 31-87), 43 (49\%) were female, $33(38 \%)$ had a carcinoid syndrome, and $12(14 \%)$ had carcinoid heart disease. Thirty-six (41\%) patients underwent a right hemicolectomy, 10 (11\%) underwent a right hemicolectomy prolonged with SB resection, and 42 (48\%) underwent SB segmental resection. The median length of SB resection was $130 \mathrm{~cm}$ (range, 15-520). The median tumor size was $18 \mathrm{~mm}$ (range, 7-70), mainly T3 (76\%) and grade 1 (66\%). Almost all (94\%) SI-NET had involved LN, with median LN ratio of 0.19 (range, $0-0.86$ ), and a median of 27 removed LN (range, 5-86); 60 (68\%; $p=0.170)$ and $34(39 \%)$ patients had liver and/or peritoneal metastases. Overall survival rate at 5 years was $84.6 \%$ (standard deviation, 5.9).

Patients with multiple tumors were significantly more often male and symptomatic than those with unifocal ileal-NET. They underwent less right hemicolectomy, median length of remaining JI was significantly shorter (445 $\mathrm{cm}$; range, 270-680) than in unifocal ileal-NET $(510 \mathrm{~cm}$; range, $210-760 ; p=0.04$ ) and median length of resected JI was significantly longer $(180 \mathrm{~cm}$; range, $40-520$ vs. 80 $\mathrm{cm}$; range, $15-360 ; p=0.001)$. Multiple tumors had more often a mesenteric mass ( 84.1 vs. $60.5 \%, p=0.014)$ but were significantly smaller in size ( 17 vs. $20 \mathrm{~mm}, p=0.049$ ). Median LNR was significantly higher in multiple tumors ( 0.28 vs. $0.12, p=0.002$ ), but other tumor characteristics (grade [grade 2, 34.9 vs. $34.1 \%, p=0.938$ ], Ki67 [median of 1.5 vs. $1.2, p=0.992$ ], lymphovascular [ 70.7 vs. $65.9 \%$, $p=0.635]$ and perineural [ 65.0 vs. $70.0 \%, p=0.633]$ invasion, $\mathrm{LN}$ involvement [ 95.5 vs. $93.2 \%, p=0.645$ ], and liver [75.0 vs. $61.4 \%, p=0.170$ ] or peritoneal [ 40.9 vs. $36.4 \%$, $p=0.661]$ metastases) and overall 5-year survival were not significantly different between multiple and unifocal tumors (survival at 5 years of 82.6 vs. $86.2 \%, p=0.975$, Table 1).

Thirty of the 44 (68\%) unifocal ileal-NET were located in the last $40 \mathrm{~cm}$ of the $\mathrm{SB}$, thus on the right side of the theoretical area of the SMA axis; the median length from the ICV was $10 \mathrm{~cm}$ (range, 0-220), significantly more distal than the last tumor in the multiple SI-NET $(103 \mathrm{~cm}$; range, $1-290 ; p<0.001)$. The median percentage of the distance between the DJA and unifocal ileal-NET was 
Table 1. Comparison of unifocal versus multiple ileal-NET

\begin{tabular}{|c|c|c|c|c|}
\hline Median age, years (range) & $66(31-87)$ & $64(31-87)$ & $66(45-82)$ & 0.135 \\
\hline Symptomatic patient, $n(\%)$ & $69(78.4)$ & $29(65.9)$ & $40(90.9)$ & 0.004 \\
\hline Carcinoid syndrome, $n(\%)$ & $33(37.5)$ & $13(29.5)$ & $20(45.5)$ & 0.123 \\
\hline Carcinoid heart disease, $n(\%)$ & $12(13.6)$ & $3(6.8)$ & $9(20.5)$ & 0.062 \\
\hline Right hemicolectomy prolonged with SB resection & $10(11.4)$ & $0(0.0)$ & $10(22.7)$ & \\
\hline SB segmental resection & $42(47.7)$ & $11(25.0)$ & $31(70.5)$ & \\
\hline Median length of JI, cm (range) & $620(310-870)$ & $620(310-870)$ & $630(480-810)$ & 0.295 \\
\hline Median length of JI resection, $\mathrm{cm}$ (range) & $130(15-520)$ & $80(15-360)$ & $180(40-520)$ & 0.001 \\
\hline Median length of remaining JI, cm (range) & $470(210-760)$ & $510(210-760)$ & $445(270-680)$ & 0.040 \\
\hline \multicolumn{5}{|l|}{ Median length, $\mathrm{cm}$ (range) of the } \\
\hline Median percentage of distance (b)/total JI length & $83.8(54.7-100)$ & $98.6(66.2-100)$ & $83.3(54.7-100)$ & $<0.001$ \\
\hline Median percentage of distance (c)/total JI length & $11.2(0.0-45.3)$ & $1.6(0.0-33.8)$ & $16.7(0.2-45.3)$ & $<0.001$ \\
\hline Median size of the largest tumor, mm (range) & $18(7-70)$ & $20(7-70)$ & $17(7-40)$ & 0.049 \\
\hline \multicolumn{5}{|l|}{ Pathological (p)T stage of the most invasive tumor, $n(\%)$} \\
\hline $\mathrm{pT} 1$ & $4(4.6)$ & $3(6.8)$ & $1(2.3)$ & 0.771 \\
\hline pT2 & $9(10.2)$ & $4(9.1)$ & $5(11.4)$ & \\
\hline pT3 & $67(76.1)$ & $32(72.7)$ & $35(79.5)$ & \\
\hline pT4 & $8(9.1)$ & $5(11.4)$ & $3(6.8)$ & \\
\hline \multicolumn{5}{|l|}{ Highest tumor grade, $n(\%)$} \\
\hline Grade 1 & $57(65.5)$ & $29(65.9)$ & $28(65.1)$ & 0.938 \\
\hline Grade 2 & $30(34.5)$ & $15(34.1)$ & $15(34.9)$ & \\
\hline Median Ki67 index, \% (range) & $1.3(0-20.0)$ & $1.2(0-20.0)$ & $1.5(0-18.0)$ & 0.992 \\
\hline Mesenteric mass, $n(\%)$ & $63(72.4)$ & $26(60.5)$ & $37(84.1)$ & 0.014 \\
\hline Liver metastases, $n(\%)$ & $60(68.2)$ & $27(61.4)$ & $33(75.0)$ & 0.170 \\
\hline Peritoneal metastases, $n(\%)$ & $34(38.6)$ & $16(36.4)$ & $18(40.9)$ & 0.661 \\
\hline Overall survival at 5 years, $\%$ (SD) & $84.6(5.9)$ & $86.2(8.0)$ & $82.6(9.1)$ & 0.975 \\
\hline
\end{tabular}

JI, jejuno-ileum; SB, small bowel; DJA, duodenojejunal angle; ICV, ileocecal valve; LN, lymph node.

98.6\% (range, 66.2-100.0) of the entire JI length (Fig. 2a, c; Table 1).

Conversely, 40 of the 44 (91\%) multiple SI-NET were located significantly more proximal than the last $40 \mathrm{~cm}$ of the SB compared to unifocal ileal-NET $(p<0.001)$, thus on the left side of the theoretical area of the SMA axis. The median distance from the DJA ranged from $428 \mathrm{~cm}$ (range, 300-635) for proximal tumors to $542 \mathrm{~cm}(350-$ $725)$ for distal tumors $(p<0.001$ and 0.168 for the most proximal and the most distal tumor, compared to unifocal SI-NET); thus, the median percentage of the distance between the DJA and the most proximal ileal-NET was $67.4 \%$ (range, $47.7-99.8$ ) of the entire JI length, and $83.3 \%$ (54.7-100.0) between the DJA and the most distal ilealNET, significantly more proximal than unifocal tumors $(p<0.001$ for both the most proximal and the most distal tumor). The median number of tumors was 7 (range, 2-95); they occurred in a median SB length of $105 \mathrm{~cm}$ 


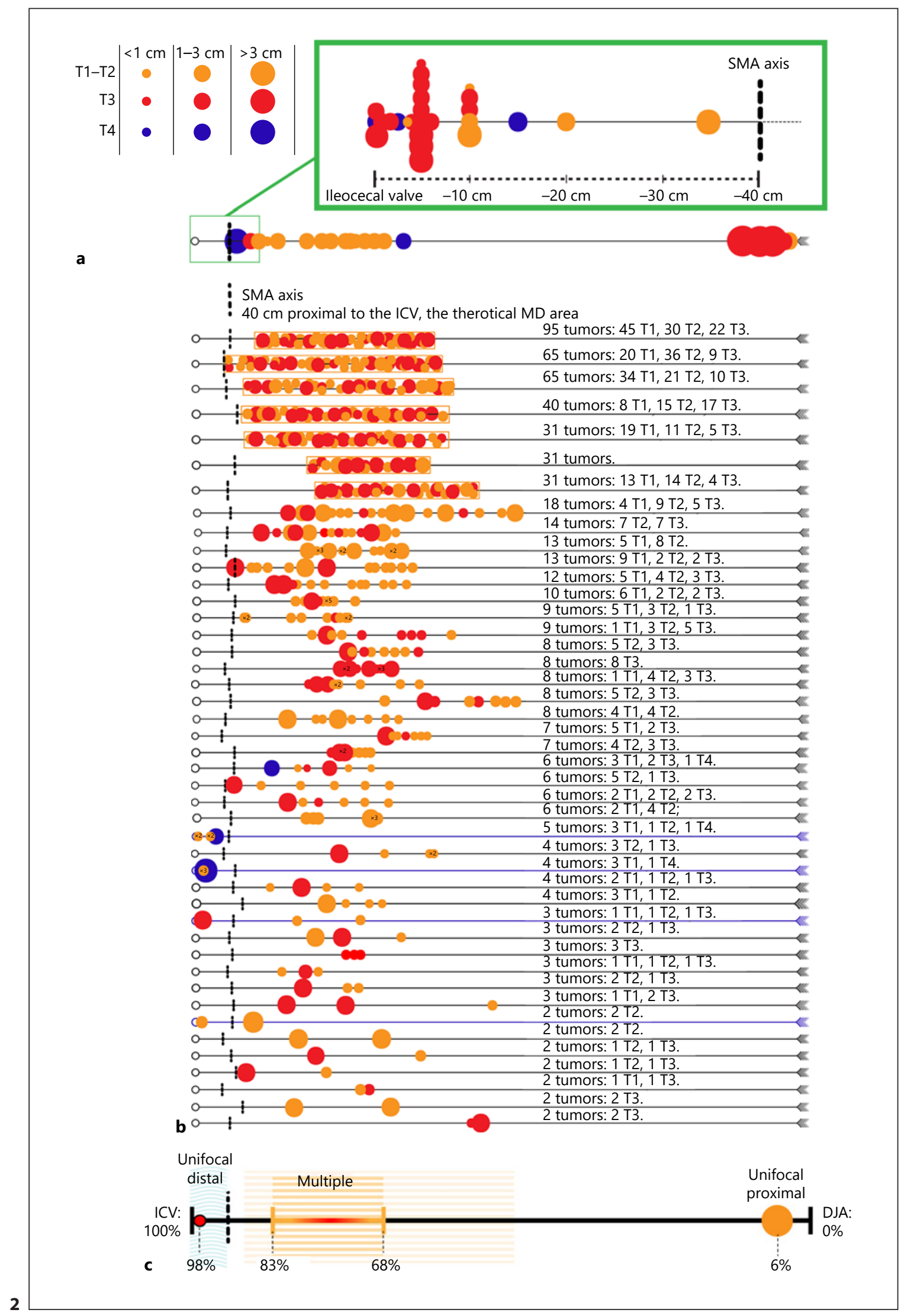

(For legend see next page.) 
(10-240), with a median interval of $10 \mathrm{~cm}(0-120$; Fig. 2b, c; Table 1).

In 6 patients, tumor count was not possible during surgery due to mesenteric sclerosis with SB sticking together. During pathological evaluation, 5 were found to be multiple and one was unifocal. In total, the surgeon counted 604 tumors and the pathologist counted 651 tumors. For 3 patients, only 1 tumor was palped, but multiple tumors were finally detected by pathologic evaluation. For 1 patient, 2 tumors were palped by the surgeon, and finally, one of these was a benign tumor from connective tissue. Concordance between surgical and pathological reports for tumor count by patient was 61/88 (69\%): 48/49 (98\%) for unifocal and 13/39 (33\%) for multiple tumors. For multiple SI-NET, the surgeon had either overestimated the tumor count (11/39 patients) and/or missed some lesions (15/39 patients).

\section{Discussion}

We show for the first time that unifocal and multiple SI-NETs have different preferred locations along the SB: about $70 \%$ of the unifocal ileal-NET were located within the very last part of the SB, while almost $91 \%$ of multiple SI-NET were more proximal, separated by the theoretical area of the SMA axis. In addition, and similarly to Keck et al. [6], we report that a careful SB palpation led to the detection of multiple SI-NET in about half of the patients, most of which were located within a SB segment of about $1 \mathrm{~m}$ in the ileum. These results underline the clinical importance of a thorough evaluation of the entire SB for multiple tumors, as mandatory by current recommendations [16]. This may prevent the occurrence of new recurrences in patients with multiple SI-NET. The lack of systematic palpation might explain the results reported by Le Roux et al. [17] in which more recurrences occurred in multiple versus unifocal SI-NET, but in which some multiple tumors may have been missed.

We report herein 3 types of SI-NET. The first group concerns very rare cases of jejunal-NET, which, as previously reported, were found to be unifocal, with a larger tumor size than ileal-NET, and without serotonin expression [18]. They mostly occurred in patients without car-

Fig. 2. Location of unifocal (a) and multiple (b) SI-NET, and the median distance of tumors from the DJA in percentage of the JI segment (c). SI-NET, small-intestinal neuroendocrine tumors; DJA, duodenojejunal angle; ICV, ileocecal valve; SMA, superior mesenteric artery; JI, jejuno-ileum.

Small-Intestinal Neuroendocrine Tumors Location cinoid syndrome and had a poorer prognosis. These rare SI-NET probably have a different carcinogenesis and will not be discussed further. Second, as previously indicated by other studies included from the Surveillance, Epidemiology, and End Results (SEER) database [1], the other 2 main tumor groups appear to be mainly located in the ileum segment, are often metastatic, and often associated with carcinoid syndrome. The present study shows that the latter 2 groups can be separated, based on location, by the theoretical SMA axis, with multiple SI-NET being more proximal than unifocal ones. In line with the present results, Keck et al. [6] reported that 77 of 107 (72\%) patients had tumors within $100 \mathrm{~cm}$ from the ICV, and that 36 of the 46 (78\%) distal tumors were unifocal versus 10 of $29(34 \%)$ for those in the mid-SB $(p<0.01)$. Poncet et al. [19] also published 8 cases of SI-NET developed in the MD, all of which were unifocal. However, no other studies reported specific locations of SI-NET according to their unifocal or multiple status.

Comparisons between unifocal and multiple SI-NET are scarce $[4,6,17]$. In the present study, we show that multiple SI-NET were more common in men, but we do not yet have an explanation for that. In 2003, Yantiss et al. [4] showed that patients with multiple SI-NET had a significantly higher risk of developing carcinoid syndrome, and had a poorer prognosis than patients with unifocal tumors. In the present study, although prognosis was not significantly different between both groups, there was a tendency for more carcinoid syndrome, as well as a higher LNR and more mesenteric mass in patients with multiple SI-NET than in those with unifocal tumors. Similarly, Keck et al. [6] found that unifocal SI-NET had larger tumor sizes than multiple SI-NET (2.3 vs. $1.6 \mathrm{~cm}$ ). Further studies are needed to understand whether there are differences in carcinogenesis between unifocal and multiple SI-NET. It has been proposed that multiple SI-NET represent "drop metastases" [20], consistent with the limited spatial distribution of the tumors, but this hypothesis does not match with the independent clonal origin of multiple tumors within a patient $[21,22]$.

There are some hypotheses to explain the propensity for tumor formation in the distal ileum, such as ileumspecific environmental factors (site of bile salt and vitamin B12 absorption, expression of glucagon-like peptide 1 , and stasis of its contents due to the braking actions of the ICV) [6]. However, the main question now concerns the preferred location of unifocal SI-NET in the very last SB segment, compared to multiple SI-NET which are more proximal although still in the ileum. One possible explanation could stem from the different embryological

Neuroendocrinology 2021;111:786-793

791 
origin and thus different anatomy between the 2 SB parts, more specifically implicating the development of the enteric nervous system (ENS). The latter represents the intrinsic innervation of the gastrointestinal tract, and, in close collaboration with endocrine cells, is implicated in controlling gastric acid secretion, changing blood flow, and SB motility [23-25]. The development of ENS follows different pathways, depending on the final destination of neural precursor cells during migration: the pre- and post-umbilical gut, which are separated by the SMA axis $[26,27]$. ENS comes from vagal and sacral neural crest cells (NCC) [28]: vagal NCC invade the foregut first (duodenum and jejunum) and finally colonize the entire intestine, while sacral NCC first colonize the hindgut (rectum and colon) which continues to the umbilicus, without ever reaching the pre-umbilical SB $[28,29]$. The main anatomical difference between the 2 sides is that on the left side of the SMA axis, vagal NCC migrated into the submucosa before colonizing the myenteric plexus region (between the circular and longitudinal layers of smooth muscle), whereas on the right side of the SMA axis, the NCC migrated next to the serosal epithelium and migrated to the submucosa secondarily [30-32]. Therefore, because SI-NET have the capacity to rapidly develop within the submucosa, SI-NET cells of the pre-umbilical SB could be more rapidly related to the ENS than to postumbilical tumors. Further studies are warranted to confirm this finding and to explore the role of ENS in SI-NET carcinogenesis, particularly in the development of multiple tumors.

This work has certain limitations. It is a retrospective study conducted in a single institution. In addition, this study was unable to fully validate the hypothesis that unifocal versus multiple ileal-NET could be separated by the SMA axis: indeed, unifocal tumors were not all located on the right side of the "MD" point. Two explanations could be proposed for this observation. The distinction between the 2 groups (unifocal vs. multiple) is based on the surgeon's and pathologist's ability to find multiple tumors. For 3 patients, only 1 tumor was palped, and others were discovered by pathologic evaluation. Thus, some "unifocal" SI-NET located on the left side of the SMA axis may be misclassified. Second and more importantly, since MD was rarely visible, we chose $40 \mathrm{~cm}$ from the ICV as the potential area for delineating the SMA axis, but this distance is variable depending on the population. Most authors have described it at a distance between 30 and $90 \mathrm{~cm}$ proximal from the ICV [7-12]. Using this $40 \mathrm{~cm}$ threshold, $30 \%$ of unifocal SI-NET were proximal to this distance. However, the MD could be much more proximal, as in this case described in 1978 with the MD being $10 \mathrm{~cm}$ distal to the ligament of Treitz [33]. These patients may therefore have an "MD" point more proximal and their unifocal ileal-NET may remain on the right side of the SMA axis.

In conclusion, multiple SI-NET are mostly located on the left side of the SMA axis and should be sought for by the surgeon including a careful palpation of the entire SB. Further studies are warranted to explore the embryological origin of unifocal versus multiple SI-NET.

\section{Acknowledgements}

We thank Verena Landel, from the DRCI (Direction de la Recherche Clinique et de l'Innovation), Hospices Civils de Lyon, for her help in writing and preparing the manuscript. We thank Pr Jean-Alain Chayvialle for its NET patients' management during many years and for his advice in writing the manuscript.

\section{Statement of Ethics}

This retrospective study was conducted according to the World Medical Association Declaration of Helsinki. The database was approved by the national data protection commission (Commission nationale de l'informatique et des libertés, CNIL) on November 6, 2015 (No. 15-111). Consent was obtained from each living patient after a full explanation of the purpose and nature of all procedures used.

\section{Conflict of Interest Statement}

The authors have no conflicts of interest to disclose.

\section{Funding Sources}

This study did not receive any specific funding from any commercial or not-for-profit public sector funding agency.

\section{Author Contributions}

Study conception and design: Maroin Kalifi, Thomas Walter, and Gilles Poncet. Acquisition of data: Maroin Kalifi, Thomas Walter, Laurent Milot, Valerie Hervieu, Colette Roche, Arnaud Pasquer, and Gilles Poncet. Analysis and interpretation of data: Maroin Kalifi, Thomas Walter, Benjamin Gibert, Colette Roche, Julien Forestier, Catherine Lombard Bohas, and Gilles Poncet. Drafting of manuscript: Maroin Kalifi, Thomas Walter, Laurent Milot, Arnaud Pasquer, and Gilles Poncet. Critical revision: All authors reviewed the manuscript before submission and agree with its contents. 


\section{References}

1 Dasari A, Shen C, Halperin D, Zhao B, Zhou $\mathrm{S}, \mathrm{Xu} \mathrm{Y}$, et al. Trends in the incidence, prevalence, and survival outcomes in patients with neuroendocrine tumors in the United States. JAMA Oncol. 2017;3(10):1335-42.

2 Gangi A, Siegel E, Barmparas G, Lo S, Jamil LH, Hendifar A, et al. Multifocality in small bowel neuroendocrine tumors. J Gastrointest Surg. 2018;22(2):303-9.

3 Strosberg JR, Weber JM, Feldman M, Coppola D, Meredith K, Kvols LK. Prognostic validity of the American Joint Committee on cancer staging classification for midgut neuroendocrine tumors. J Clin Oncol. 2013; 31(4):420-5.

4 Yantiss RK, Odze RD, Farraye FA, Rosenberg AE. Solitary versus multiple carcinoid tumors of the ileum: a clinical and pathologic review of 68 cases. Am J Surg Pathol. 2003;27(6): 811-7.

5 Pasquer A, Walter T, Hervieu V, Forestier J, Scoazec JY, Lombard-Bohas C, et al. Surgical management of small bowel neuroendocrine tumors: specific requirements and their impact on staging and prognosis. Ann Surg Oncol. 2015;22(S3):S742-9.

6 Keck KJ, Maxwell JE, Utria AF, Bellizzi AM, Dillon JS, O'Dorisio TM, et al. The distal predilection of small bowel neuroendocrine tumors. Ann Surg Oncol. 2018;25(11):3207-13.

7 Carlioz P. Le diverticule de Meckel, de l'embryologie à la chirurgie. e-Mémoires de l'Académie Nationale de Chirurgie [serial online]. 2014;13(2):001-6.

8 Nafisa KK, Yvan JS, Venkata SK, Mario RV, Azmath M. Meckel diverticulum: background, anomalies of omphalomesenteric duct, complications of Meckel diverticulum. Medscape [serial online]. 2019 Nov.

9 Hansen CC, Søreide K. Systematic review of epidemiology, presentation, and management of Meckel's diverticulum in the 21st century. Medicine. 2018;97(35):e12154.

10 Martin JP, Connor PD, Charles K. Meckel's diverticulum. Am Fam Physician. 2000;61(4): 1037-42.

11 Miltiadis IM, Michalis F, Bassilis K, Athanasios DG. Incidence, complications, and management of Meckel's diverticulum. Arch Surg. 1995;130(2):143-6.
12 Yamaguchi M, Takeuchi S, Awazu S. Meckel's diverticulum. Am J Surg. 1978;136(2):247-9.

13 Pasquer A, Poncet G. Small bowel neuroendocrine tumors surgery: technical point: with video. J Visc Surg. 2017;154(1):61-2.

14 Pasquer A, Walter T, Rousset P, Hervieu V, Forestier J, Lombard-Bohas C, et al. Lymphadenectomy during small bowel neuroendocrine tumor surgery: the concept of skip metastases. Ann Surg Oncol. 2016;23(Suppl 5): 804-8.

15 Lardière-Deguelte S, de Mestier L, Appéré F, Vullierme MP, Zappa M, Hoeffel C, et al. Toward a preoperative classification of lymph node metastases in patients with small intestinal neuroendocrine tumors in the era of intestinal-sparing surgery. Neuroendocrinology. 2016;103(5):552-9.

16 De Mestier L, Lepage C, Baudin E, Coriat R, Courbon F, Couvelard A, et al. Digestive neuroendocrine neoplasms (NEN): French intergroup clinical practice guidelines for diagnosis, treatment and follow-up (SNFGE, GTE, RENATEN, TENPATH, FFCD, GERCOR, UNICANCER, SFCD, SFED, SFRO, SFR). Dig Liver Dis. 2020 Mar;52(5):473-92.

17 Le Roux C, Lombard-Bohas C, Delmas C, Dominguez-Tinajero S, Ruszniewski P, Samalin E, et al. Relapse factors for ileal neuroendocrine tumours after curative surgery: a retrospective French multicentre study. Dig Liver Dis. 2011;43(10):828-33.

18 Chopin-Laly X, Walter T, Hervieu V, Poncet G, Adham M, Guibal A, et al. Neuroendocrine neoplasms of the jejunum: a heterogeneous group with distinctive proximal and distal subsets. Virchows Arch. 2013;462(5):489-99.

19 Poncet G, Hervieu V, Walter T, Lépinasse F, Chardon L, Pilleul F, et al. Neuroendocrine tumors of Meckel's diverticulum: lessons from a single institution study of eight cases. J Gastrointest Surg. 2011;15(1):101-9.

20 Wang YZ, Carrasquillo JP, McCord E, Vidrine R, Lobo ML, Zamin SA, et al. Reappraisal of lymphatic mapping for midgut neuroendocrine patients undergoing cytoreductive surgery. Surgery. 2014;156(6):1498-3.

21 Zhang Z, Mäkinen N, Kasai Y, Kim GE, Diosdado B, Nakakura E, et al. Patterns of chromosome 18 loss of heterozygosity in multifocal ileal neuroendocrine tumors. Genes Chromosomes Cancer. 2020;59(9):535-9.
22 Katona TM, Jones TD, Wang M, AbdulKarim FW, Cummings OW, Cheng L. Molecular evidence for independent origin of multifocal neuroendocrine tumors of the enteropancreatic axis. Cancer Res. 2006;66(9): 4936-42.

23 Furness JB, Callaghan BP, Rivera LR, Cho HJ. The enteric nervous system and gastrointestinal innervation: integrated local and central control. Adv Exp Med Biol. 2014;817:39-71.

24 Furness JB. Integrated neural and endocrine control of gastrointestinal function. Adv Exp Med Biol. 2016;891:159-73.

25 Uesaka T, Young HM, Pachnis V, Enomoto $\mathrm{H}$. Development of the intrinsic and extrinsic innervation of the gut. Dev Biol. 2016;417(2): 158-67.

26 Young HM, Hearn CJ, Newgreen DF. Embryology and development of the enteric nervous system. Gut. 2000;47(90004):iv12-4.

27 Rao M, Gershon MD. Enteric nervous system development: what could possibly go wrong? Nat Rev Neurosci. 2018;19(9):552-65.

28 Burns AJ, Douarin NM. The sacral neural crest contributes neurons and glia to the postumbilical gut: spatiotemporal analysis of the development of the enteric nervous system. Development. 1998;125(21):4335-47.

29 Douarin NML, Teillet M-A. The migration of neural crest cells to the wall of the digestive tract in avian embryo. Development. 1973; 30(1):31-48

30 Rothman TP, Le Douarin NM, FontainePérus JC, Gershon MD. Colonization of the bowel by neural crest-derived cells re-migrating from foregut backtransplanted to vagal or sacral regions of host embryos. Dev Dyn. 1993;196(3):217-33.

31 Burns AJ, Le Douarin NM. Enteric nervous system development: analysis of the selective developmental potentialities of vagal and sacral neural crest cells using quail-chick chimeras. Anat Rec. 2001;262(1):16-28.

32 Wang X, Chan AK, Sham MH, Burns AJ, Chan WY. Analysis of the sacral neural crest cell contribution to the hindgut enteric nervous system in the mouse embryo. Gastroenterology. 2011;141(3):992-6.

33 McSwain GR, Anderson MC. Meckel's diverticulum of the proximal jejunum. Arch Surg. $1979 ; 114(2): 212$. 